Supplement of Biogeosciences, 15, 2091-2109, 2018

https://doi.org/10.5194/bg-15-2091-2018-supplement

(C) Author(s) 2018. This work is distributed under

the Creative Commons Attribution 4.0 License.

(c) (1)

Supplement of

\title{
Biogeochemical characteristics of suspended particulate matter in deep chlorophyll maximum layers in the southern East China Sea
}

Qianqian Liu et al.

Correspondence to: Selvaraj Kandasamy (selvaraj@xmu.edu.cn)

The copyright of individual parts of the supplement might differ from the CC BY 4.0 License. 


\section{Supplement}

Table S1. Hydrographic parameters (temperature, salinity and turbidity), chlorophyll fluorescence concentration and elemental (POC, $\mathrm{PN})$ and isotopic $\left(\delta^{13} \mathrm{C}_{\mathrm{POC}}\right.$ and $\delta^{15} \mathrm{NPN}$ ) compositions of particulate organic matter around the water depths of deep chlorophyll maximum in the southern East China Sea. Chl a is the converted value using the linear relationship between measured $\mathrm{Chl} a$ and $\mathrm{Chl}$ fluorescence obtained using in situ fluorometer onboard.

\begin{tabular}{|c|c|c|c|c|c|c|c|c|c|c|c|c|c|}
\hline Station & $\begin{array}{l}\text { Long. } \\
\left({ }^{\circ} \mathrm{E}\right)\end{array}$ & $\begin{array}{l}\text { Lat. } \\
\left({ }^{\circ} \mathrm{N}\right)\end{array}$ & $\begin{array}{l}\text { Sampling } \\
\text { Depth } \\
\text { (m) }\end{array}$ & $\begin{array}{c}\text { Temp. } \\
\left({ }^{\circ} \mathrm{C}\right)\end{array}$ & Sal. & $\begin{array}{c}\text { Tur. } \\
\text { (FTU) }\end{array}$ & $\begin{array}{c}\text { Chl } \\
\text { Fluorescence } \\
\left(\mu g L^{-1}\right)\end{array}$ & $\begin{array}{c}P O C \\
\left(\mu g L^{-1}\right)\end{array}$ & $\begin{array}{c}P N \\
\left(\mu g L^{-1}\right)\end{array}$ & $\begin{array}{c}\delta^{13} \mathrm{CPOC}_{\text {POC }} \\
(\% 0)\end{array}$ & $\begin{array}{c}15 \mathrm{~N}_{\mathrm{PN}} \\
(\% 0)\end{array}$ & $\begin{array}{c}\mathrm{C} / \mathrm{N} \\
\text { Molar }\end{array}$ & $\begin{array}{c}\mathrm{POC} / \mathrm{Chl} a \\
\left(\mathrm{~g} \mathrm{~g}^{-1}\right)\end{array}$ \\
\hline $\mathrm{DH} 1-1$ & 123.00 & 30.00 & 20 & 20.26 & 33.64 & 0.46 & 0.68 & 98.4 & 19.3 & -20.7 & 5.8 & 5.9 & 144.6 \\
\hline $\mathrm{DH} 2-1$ & 123.10 & 29.47 & 20 & 22.09 & 33.92 & 0.18 & 1.12 & 110.2 & 24.5 & -21.9 & 6.3 & 5.3 & 111.1 \\
\hline $\mathrm{DH} 2-2$ & 123.40 & 29.32 & 30 & 20.16 & 33.96 & 0.19 & 0.37 & 67.4 & 13.2 & -22.3 & 6.0 & 6.0 & 147.4 \\
\hline CON02 & 122.15 & 28.50 & 20 & 19.33 & 32.70 & 4.75 & 0.15 & 92.6 & 20.5 & -20.7 & 8.0 & 5.3 & 303.3 \\
\hline $\mathrm{DH} 3-2$ & 122.80 & 28.80 & 30 & 26.14 & 33.60 & 1.07 & 0.68 & 98.8 & 20.0 & -22.9 & 6.6 & 5.8 & 145.1 \\
\hline DH3-3 & 123.20 & 28.60 & 50 & 26.72 & 33.78 & 0.12 & 0.75 & 71.5 & 15.3 & -23.7 & 6.9 & 5.4 & 98.3 \\
\hline $\mathrm{DH} 3-4$ & 123.50 & 28.40 & 50 & 27.26 & 33.68 & 0.14 & 1.39 & 72.2 & 13.5 & -23.5 & 5.8 & 6.2 & 60.9 \\
\hline $\mathrm{DH} 4-3$ & 122.80 & 28.04 & 30 & 28.20 & 33.58 & 0.08 & 0.37 & 49.8 & 9.7 & -23.8 & 6.1 & 6.0 & 108.7 \\
\hline $\mathrm{DH} 4-4$ & 123.21 & 27.78 & 50 & 26.50 & 33.75 & 0.24 & 1.23 & 82.4 & 17.4 & -22.8 & 6.1 & 5.5 & 77.0 \\
\hline DH5-1 & 121.90 & 27.80 & 30 & 25.47 & 33.79 & 3.45 & 4.07 & 263.0 & 52.8 & -18.2 & 6.0 & 5.8 & 85.5 \\
\hline DH5-5 & 123.00 & 27.13 & 50 & 21.91 & 34.31 & 0.14 & 0.81 & 49.0 & 10.1 & -23.0 & 5.8 & 5.6 & 63.4 \\
\hline DH5-6 & 123.20 & 26.96 & 50 & 22.90 & 34.50 & 0.25 & 1.62 & 81.9 & 16.0 & -23.4 & 4.8 & 6.0 & 61.0 \\
\hline
\end{tabular}




\begin{tabular}{|c|c|c|c|c|c|c|c|c|c|c|c|c|c|}
\hline DH5-7 & 123.50 & 26.80 & 50 & 23.00 & 34.21 & 0.21 & 2.66 & 69.3 & 13.6 & -23.6 & 4.7 & 5.9 & 33.3 \\
\hline DH5-8 & 123.81 & 26.63 & 70 & 22.16 & 34.52 & 0.12 & 0.72 & 44.2 & 10.3 & -24.2 & 5.3 & 5.0 & 62.2 \\
\hline DH5-9 & 124.06 & 26.44 & 50 & 24.58 & 34.44 & 0.17 & 0.89 & 70.0 & 14.5 & -23.7 & 5.9 & 5.6 & 84.4 \\
\hline $\mathrm{DH} 6-1$ & 121.20 & 27.38 & 10 & 25.52 & 33.77 & No data & No data & 225.0 & 46.6 & -21.2 & 7.6 & 5.6 & No data \\
\hline DH6-3 & 121.79 & 26.99 & 50 & 26.84 & 33.74 & 0.45 & 1.19 & 98.4 & 18.1 & -22.8 & 5.9 & 6.3 & 94.7 \\
\hline $\mathrm{DH} 6-4$ & 122.20 & 26.79 & 50 & 27.38 & 33.55 & 0.13 & 1.08 & 54.0 & 14.7 & -24.6 & 7.1 & 4.3 & 56.0 \\
\hline DH6-5 & 122.50 & 26.59 & 50 & 21.71 & 34.20 & 0.30 & 1.37 & 84.2 & 15.7 & -24.2 & 3.8 & 6.3 & 72.1 \\
\hline DH6-7 & 123.20 & 26.20 & 50 & 20.67 & 34.56 & 0.09 & 0.64 & 54.4 & 11.5 & -22.7 & 6.0 & 5.5 & 83.7 \\
\hline $\mathrm{DH} 7-1$ & 120.80 & 26.76 & 20 & 24.39 & 33.92 & 5.53 & 0.75 & 130.8 & 27.6 & -21.6 & 7.8 & 5.5 & 179.2 \\
\hline DH7-2 & 121.11 & 26.58 & 30 & 26.39 & 33.80 & 0.49 & 2.49 & 104.6 & 19.9 & -24.6 & 5.1 & 6.1 & 53.3 \\
\hline $\mathrm{DH} 7-3$ & 121.50 & 26.40 & 50 & 27.55 & 33.70 & 0.31 & 1.14 & 60.4 & 12.4 & -24.6 & 5.6 & 5.7 & 60.2 \\
\hline $\mathrm{DH} 7-4$ & 121.80 & 26.23 & 50 & 26.12 & 33.75 & 0.18 & 0.77 & 69.5 & 14.3 & -23.2 & 4.5 & 5.7 & 93.4 \\
\hline $\mathrm{DH} 7-5$ & 122.10 & 26.05 & 50 & 22.41 & 34.23 & 0.45 & 3.15 & 118.8 & 22.0 & -22.7 & 5.3 & 6.3 & 48.9 \\
\hline DH7-9 & 123.20 & 25.34 & 130 & 21.70 & 34.68 & 0.04 & 0.11 & 20.4 & 4.4 & -25.2 & 7.8 & 5.4 & 73.8 \\
\hline
\end{tabular}




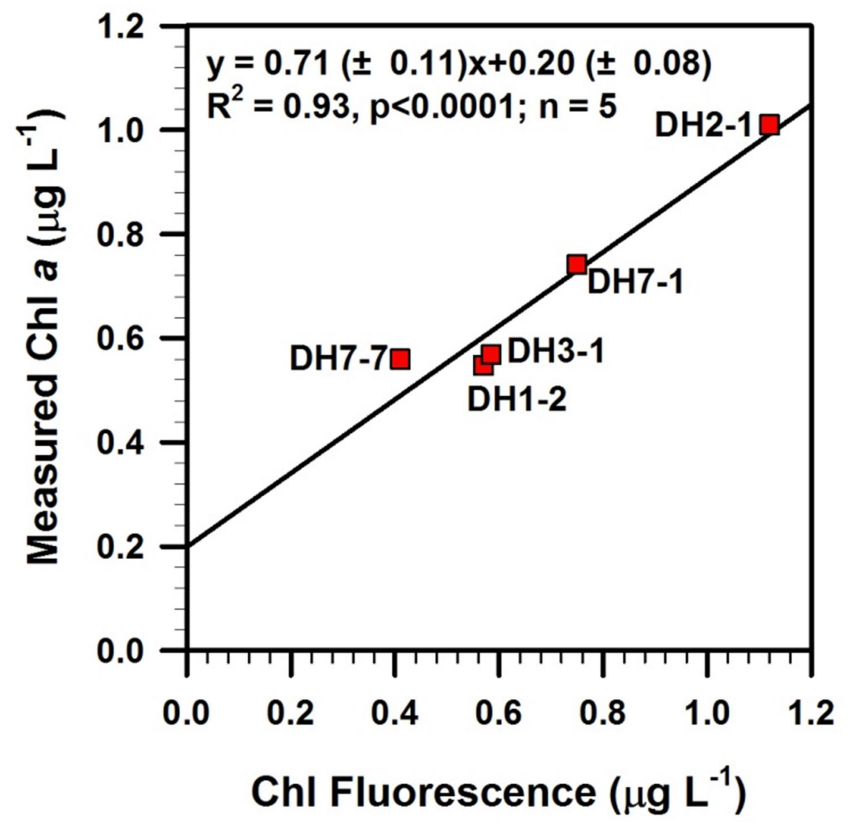

Figure S1. The relationship between the concentration of $\mathrm{Chl}$ a measured in the laboratory and the chlorophyll fluorescence values (Chl Fluorescence) obtained using in situ fluorometer onboard. A significant positive correlation between these two values in five suspended particulate matters around the water depths of deep chlorophyll maximum in the southern East China Sea was used to convert the fluorescence values to $\mathrm{Chl} a$ in this study. 